\title{
Seismic response of nonstructural components in case of nonlinear structures based on floor response spectra method
}

\author{
Marcelo Oropeza · Pascale Favez • Pierino Lestuzzi
}

Received: 21 May 2008 / Accepted: 28 June 2009 / Published online: 16 July 2009

(C) Springer Science+Business Media B.V. 2009

\begin{abstract}
This paper investigates the response of nonstructural components in the presence of nonlinear behavior of the primary structure using floor response spectra method (FRS). The effect of several parameters such as initial natural frequency of the primary structure, natural frequency of the nonstructural components (subsystem), strength reduction factor and hysteretic model have been studied. A database of 164 registered ground acceleration time histories from the European Strong-Motion Database is used. Results are presented in terms of amplification factor and resonance factor. Amplification factor quantifies the effect of inelastic deformations of the primary structure on subsystem response. Resonance factor quantifies the variation of the subsystem response considering the primary structure acceleration. Obtained results differed from precedent studies, particularly for higher primary structure periods. Values of amplification factor are improved. Obtained results of resonance factor highlight an underestimation of peak values according to current design codes such as Eurocode 8 . Therefore a new formulation is proposed.
\end{abstract}

Keywords Nonlinear seismic behavior - Nonstructural components · Floor response spectra - Resonance factor - Amplification factor - Nonlinear time history analysis . Hysteretic model $\cdot$ Recorded earthquake ground motions

\section{Introduction}

Nonstructural components or subsystem may be subject to large dynamic forces when the structure experiences earthquake ground motions. Often, an important percentage of the total cost of damage belongs to the damage on nonstructural components. This situation means that life safety is compromised if nonstructural components fail. To improve life safety and to reduce cost of damage, a better understanding of structural and nonstructural subsystems is essential.

M. Oropeza $(\varangle) \cdot$ P. Favez $\cdot$ P. Lestuzzi

IIC-Civil Engineering Institute, EPFL-École Polytechnique Fédérale de Lausanne,

ENAC-IIC-ENAC Bâtiment GC, Station 18, 1015 Lausanne, Switzerland

e-mail: marcelo.oropezaancieta@epfl.ch 
Depending on how the loads are applied, the subsystems can be classified into 2 categories:

Deformation sensitive subsystems In this case, loads are mainly due to the deformation imposed on the primary structure.

Acceleration sensitive subsystems In this case, loads are mainly due to the accelerations imposed at their attachment points. This category is used in this study.

Current construction codes, such as Eurocodes, propose guidelines for subsystem design. Eurocode 8 considers a height factor and a resonance factor (European Committee for Standardisation (CEN) 2003).

Several studies have investigated the behavior and the sensitivity of subsystems during a seismic event (see Lin and Mahin 1985; Chen and Soong 1988; Kingston 2004; Chaudhuri and Villaverde 2008). These studies propose some guidelines based on parametric studies. The investigated parameters were also used in precedent studies.

The Floor Response Spectrum method (FRS) is a common approach based on time history analysis. Results obtained with this method may perform design guidelines (Lin and Mahin 1985).

Recent studies diverge from results obtained by Lin and Mahin (Triou 2006). New values for the amplification factor considering several parameters are proposed in this paper.

Results are expressed in terms of resonance factor and compared to Eurocode 8 (European Committee for Standardisation (CEN) 2003). Finally a new formulation is proposed.

Resonance factor For seismic design of nonstructural components, Eurocodes (European Committee for Standardisation (CEN) 2003) propose an horizontal force which depends on several factors such as seismic zone, soil conditions and structural characteristics and a resonance factor that depends on the relative height of the system and the subsystem.

Amplification factor In order to quantify the effects of inelastic deformations of the primary structure on subsystem response, Lin and Mahin (1985) defined an amplification factor based on statistical evaluation. This factor is defined as the variation of ratio between the floor response spectral value for an inelastic structure and the corresponding value for an elastic structure. FRS (floor response spectra) considering inelastic behavior can be obtained from a linear elastic analysis. Lin and Mahin present their results in an investigation to be developed in further studies.

Results obtained by Lin and Mahin are based on:

- 10 ground motions from 5 earthquakes;

- 2 hysteretic models (elastoplastic model and stiffness degrading model) with no post-yield stiffness;

- several values of primary structure period, subsystem period, damping and strength reduction factor.

The amplification factor is plotted as the variation of the amplification factor versus the initial natural subsystem period (Lin and Mahin 1985).

In this study, nonlinear behavior of the primary structure is represented by the strength reduction factor. The strength reduction factor represents the capacity for a given structure to support a seismic demand, during which structure may yield. Therefore, it may be related to the displacement ductility of the structure using the well-established empirical rule of equal displacements (Fig. 1).

The equal displacement rule was found to be generally correct and almost independent of the hysteretic model for both real and synthetic earthquakes and for structures with initial natural frequencies below a frequency limit (generally between 1.5 and $2 \mathrm{~Hz}$; Lestuzzi and Badoux 2003a). 


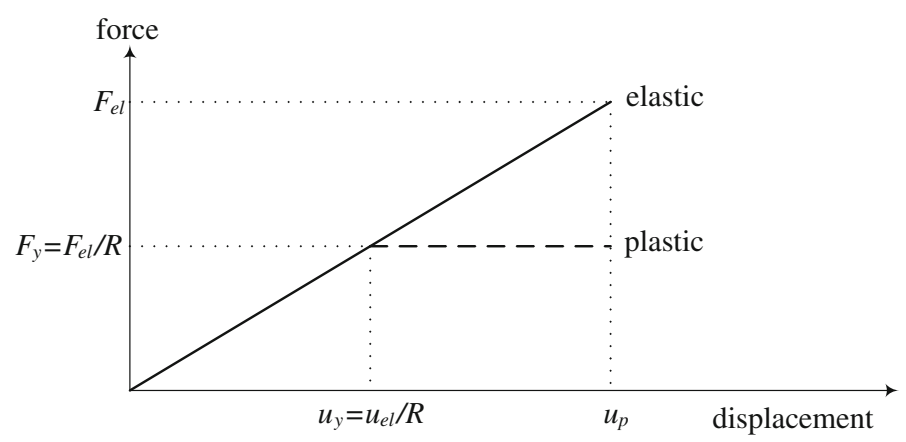

Fig. 1 The equal displacement rule compares displacements between an elastic structure and an ideal elastoplastic structure

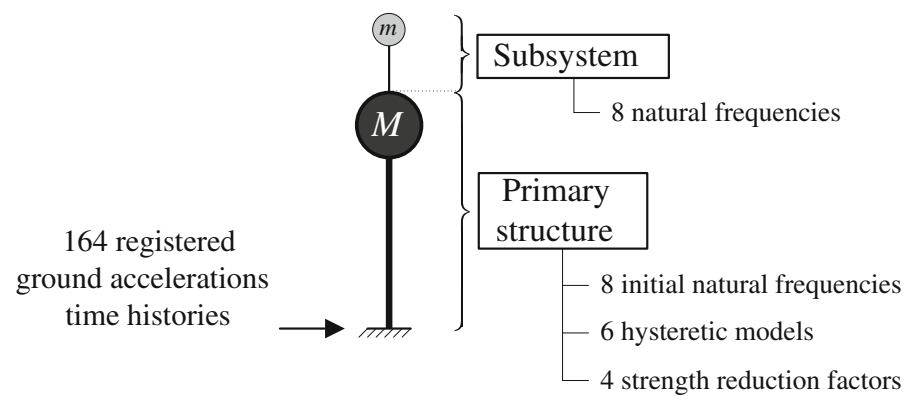

Fig. 2 Diagram showing the primary structure and the subsystem and the different cases analyzed in this paper

The strength reduction factor $R$ considers that plastic behavior of the structure has a positive impact on the earthquake energy dissipation. A weaker structure does not require a larger displacement, but a larger ductility is necessary.

\subsection{Objective}

The objective of this paper is to investigate the seismic behavior of subsystems supported on primary structures that can develop inelastic deformations and to extend obtained results in terms of amplification factor and resonance factor for design purposes.

Parameters such as initial natural frequency of the primary structure $\left(f_{\mathrm{p}}\right)$, natural frequency of the subsystem $\left(f_{\mathrm{s}}\right)$ and strength reduction factor $(R)$ are considered in order to carry out a parametric study. Six hysteretic models are studied and a database of 164 registered ground acceleration time histories from the European Strong-Motion Data Base is used (Favez 2007) (Fig. 2). Obtained results are compared to those proposed in literature in terms of resonance factor and amplification factor.

\subsection{Basic assumptions}

The results reported in this paper are valid for the following conditions:

- Subsystem mass is negligible compared to the structure's mass. The FRS is theoretically correct only when subsystem mass tends to zero (light subsystems). 
- Both the subsystem and the primary structure are modelled as single-degree-of-freedom systems.

- A viscous damping ratio with the usual value of $5 \%$ is used.

- Ductility of the structure is expressed using the strength reduction factor.

- The subsystem remains elastic and the primary structure can develop inelastic behavior.

\section{Parameters}

The primary structure is defined by 2 parameters: strength reduction factor $R$ and initial natural frequency $f_{\mathrm{p}}$. The subsystem parameter is its natural frequency, $f_{\mathrm{s}}$. The subsystem remains elastic.

Seismic response is investigated through 6 different hysteretic models:

- elastoplastic model (EP-model);

- $\quad \gamma$-model (EPG-model);

- modified Takeda-model (Tak and TakZ);

- Q-Model (Q and QZ)

A database of 164 registered ground acceleration time histories from the European StrongMotion Database is used.

\subsection{Strength reduction factor}

In this study, the values of strength reduction factors of primary structure are $R=2,3$, 4 and 5 .

\subsection{Natural frequency}

Natural frequency is one of the most important parameters of the seismic response. For the primary structure, the initial natural frequencies are $f_{\mathrm{p}}=0.25,0.5,0.75,1,1.5,2,3$ and $4 \mathrm{~Hz}$. For the subsystem, $f_{s}=0.25,0.5,1,2,3,4,6$ and $8 \mathrm{~Hz}$ are used.

\subsection{Hysteretic models}

The following hysteretic models are used:

Elastoplastic model (EP-model) The elastoplastic model is sometimes also called bilinear model. Even if it is mainly intended for elastoplastic materials, such as steel, this model is extensively used for all types of materials due to its simplicity. It is included in this study because it is often considered as a reference model in numerical simulations.

The force-displacement relationship of the EP-model (Fig. 3) is specified using only three parameters: the initial stiffness, $K$, the yield displacement, $x_{\mathrm{e}}$, and the post-yield stiffness expressed as a portion of the initial stiffness, $r K$. The value $r=5 \%$ is used in this study.

$\gamma$-model (EPG-model) The gamma model (Lestuzzi and Badoux 2003b) is a simple model developed from EP-model for a better simulation of reinforced concrete behavior. The reloading curves in the EP-model are modified by a supplementary parameter $\gamma$. For large yield excursions (displacements greater than current peak displacement), the reloading curves cross the elastic portion of the envelope at a height of $1-\gamma$ of the yield force $F_{\mathrm{y}}$. For displacements smaller than current peak displacement, the reloading curves tend to the current peak 


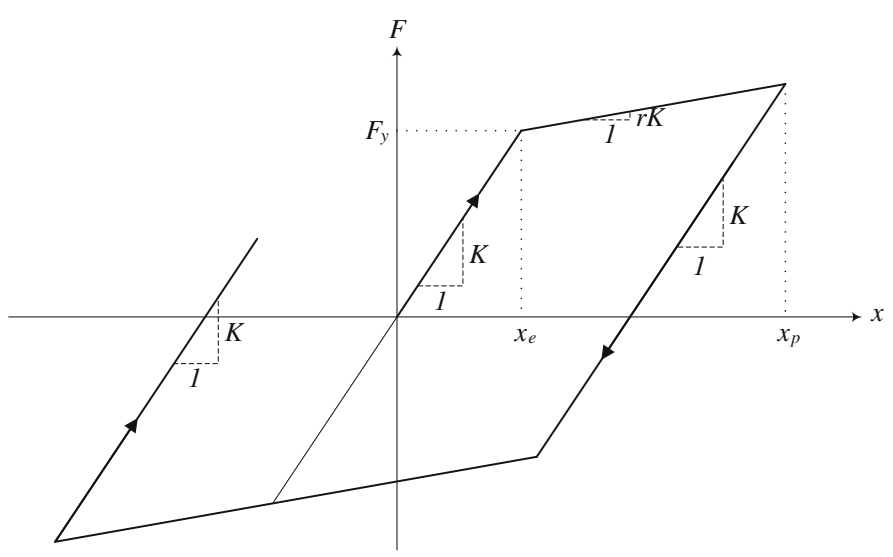

Fig. 3 Force-displacement relationship of the EP-model

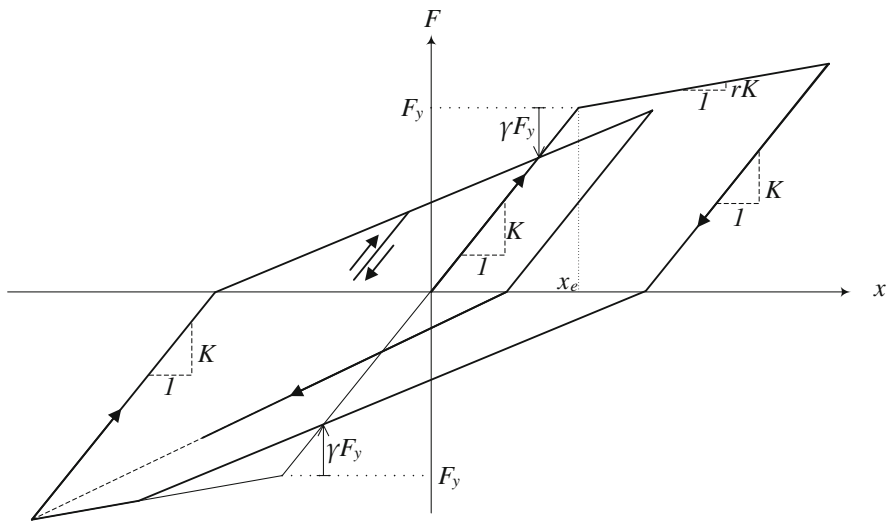

Fig. 4 Force-displacement relationship of the $\mathrm{EP} \gamma$-model

displacement. The force-displacement relationships of the $\gamma$-model are specified through four parameters: the stiffness $K$, the yield displacement $x_{\mathrm{e}}$, the post-yield stiffness $r K$, and $\gamma$ (Fig. 4). In this study $\gamma=0.35$ and $r=5 \%$ are used.

Modified Takeda-model The modified Takeda-model provides a much better simulation of the features of materials such as reinforced concrete than the EP-model. Specifically, the modified Takeda-model includes realistic conditions for the reloading curves and takes into account the degradation of the stiffness due to increasing damage, which is an important consequence when reinforced concrete is subject to seismic loading (Saatcioglu 1991). However, the modified Takeda-model does not account for strength degradation. The Takeda-model was initially proposed in an original version by Takeda et al. (1970). The modified Takeda-model was developed independently by Otani (1974) and Litton (1975). The modified Takeda-model was later adapted by many researchers. The version used here is the one of Allahabadi (Allahabadi and Powell 1988). The force-displacement relationship of the modified Takeda-model is specified through five parameters: the initial stiffness $K_{0}$, the yield displacement $x_{\mathrm{e}}$, the post yield stiffness $r K_{0}$, a parameter related to the stiffness degradation 


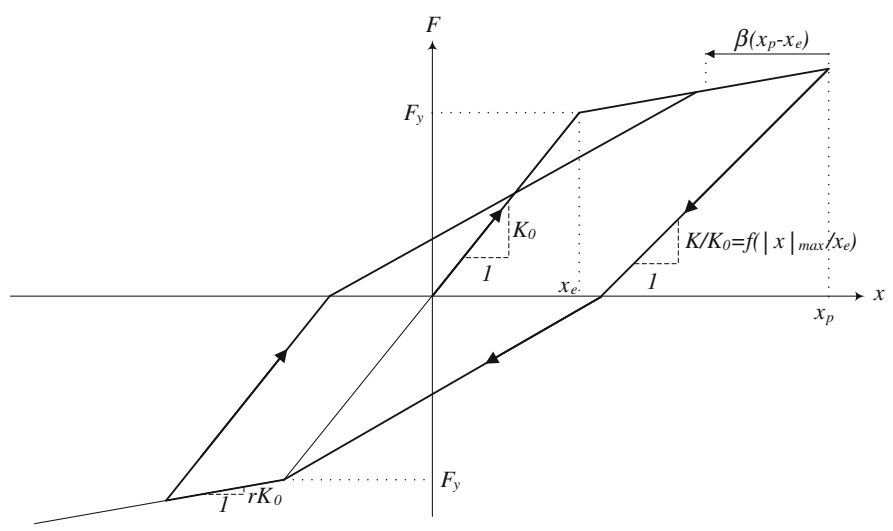

Fig. 5 Force displacement relationship of the Takeda-model

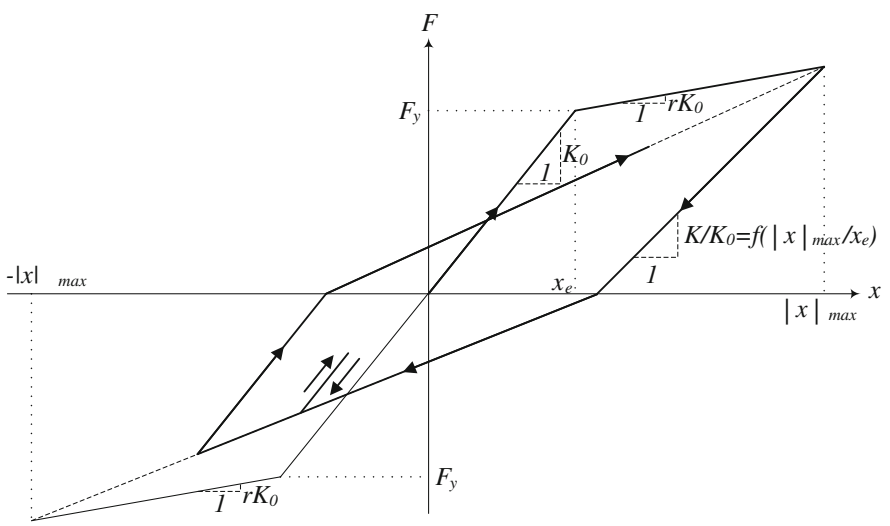

Fig. 6 Force displacement relationship of the Q-model

$\alpha$, and a parameter $\beta$, specifying the target for the reloading curve (Fig. 5). $r=5 \%$ is used in this study.

Two variants of this model are considered : $\alpha=0$ and $\beta=0$ (without stiffness degradation or TakZ) and $\alpha=0.4$ and $\beta=0$ (with stiffness degradation or Tak).

$Q$-Model The Q-model is a simplified version of the Takeda-model (Saiidi and Sozen 1981). The consideration of the absolute value of peak displacement for both directions constitutes the main simplification. The reloading curves systematically target the point corresponding to the absolute value of actual peak displacement $|x|_{\max }$.

Similar to the Takeda-model, the Q-model takes into account the stiffness degradation, but does not take into account the strength degradation. The force-displacement relationships of the Q-model are totally specified through four parameters: the initial stiffness $K_{0}$, the yield displacement $x_{\mathrm{e}}$, the post yield stiffness $r K_{0}$, and $\alpha$ related to the stiffness degradation (Fig. 6). $r=5 \%$ is used in this study.

Two variants of this model are considered: $\alpha=0$ (without stiffness degradation or QZ) and $\alpha=0.4$ (with stiffness degradation or $\mathrm{Q}$ ). 


\subsection{Ground motions}

Synthetic earthquakes may induce some bias due to their additional uncertainties. Then, nonlinear seismic behavior may be strongly influenced by synthetic earthquakes. Indeed, the use of synthetic earthquakes generally leads to an underestimation of the ductility demand (Schwab and Lestuzzi 2007). A set of 164 registered ground acceleration time histories from the European Strong-Motion Database (Ambraseys et al. 2002), selected into a more important database, is used for analysis. A set of criteria were considered in order to select earthquakes with a magnitude larger than 5 (Lestuzzi et al. 2007).

\section{Approach}

\subsection{Methodology}

Often, subsystem-primary structure interaction can be neglected and therefore analysis of subsystem response can be simplified (uncoupled analysis). Hence computation of subsystem response can be simplified. In this simplified analysis, the floor response spectrum method (FRS) is a common approach to obtain the total acceleration at the subsystem attachment point. Primary structure has a response when it is subjected to a ground motion. This response of primary structure, expressed as a relative acceleration $\left(a_{\mathrm{p}}\right)$ is then added to ground acceleration $\left(a_{\mathrm{g}}\right)$ to obtain the total acceleration of primary structure $\left(a_{\mathrm{tot}, \mathrm{p}}\right)$ and introduced as input to the subsystem. The latter has another response (FRS) expressed as relative acceleration, $a_{\mathrm{s}}$ (or as total acceleration, $a_{\mathrm{tot}, \mathrm{s}}$ ).

The differential equation of motion is computed with the central difference method, an explicit numerical method (Chopra 2001).

A program is developed to calculate the maximum acceleration in subsystem for different values of parameters (Sect. 2). In this program three steps are :

1. Primary structure response calculation

Input : 164 registered ground acceleration time histories (ground acceleration $a_{\mathrm{g}}$ vs. time) from the European Strong-Motion Database (Fig. 7a).

Output : displacement, velocity, relative acceleration, total acceleration and absolute maximum total acceleration at the top of primary structure for every combination of values of strength reduction factor, initial natural frequency, hysteretic model of primary structure and registered ground acceleration. Figure $7 \mathrm{~b}$ shows the output as a plot of relative acceleration at the top $\left(a_{\mathrm{p}}\right)$ vs. time.

2. Subsystem response calculation

Input: output of primary structure calculation in terms of total acceleration at the top $\left(a_{\mathrm{tot}, \mathrm{p}}=a_{\mathrm{p}}+a_{\mathrm{g}}\right)$. This acceleration is considered as a ground motion applied to the subsystem (Fig. 7c).

Output: relative acceleration, total acceleration (response) and absolute maximum total acceleration of the subsystem for each different value of natural frequency, for each hysteretic model of the primary structure and for each registered ground acceleration. Figure $7 d$ shows the output as a plot of relative acceleration at the top of subsystem $\left(a_{\mathrm{s}}\right)$ vs. time.

3. Output of results

The amount of intermediate results is significant. Hence, the results provided by the program permit a display of the results. 


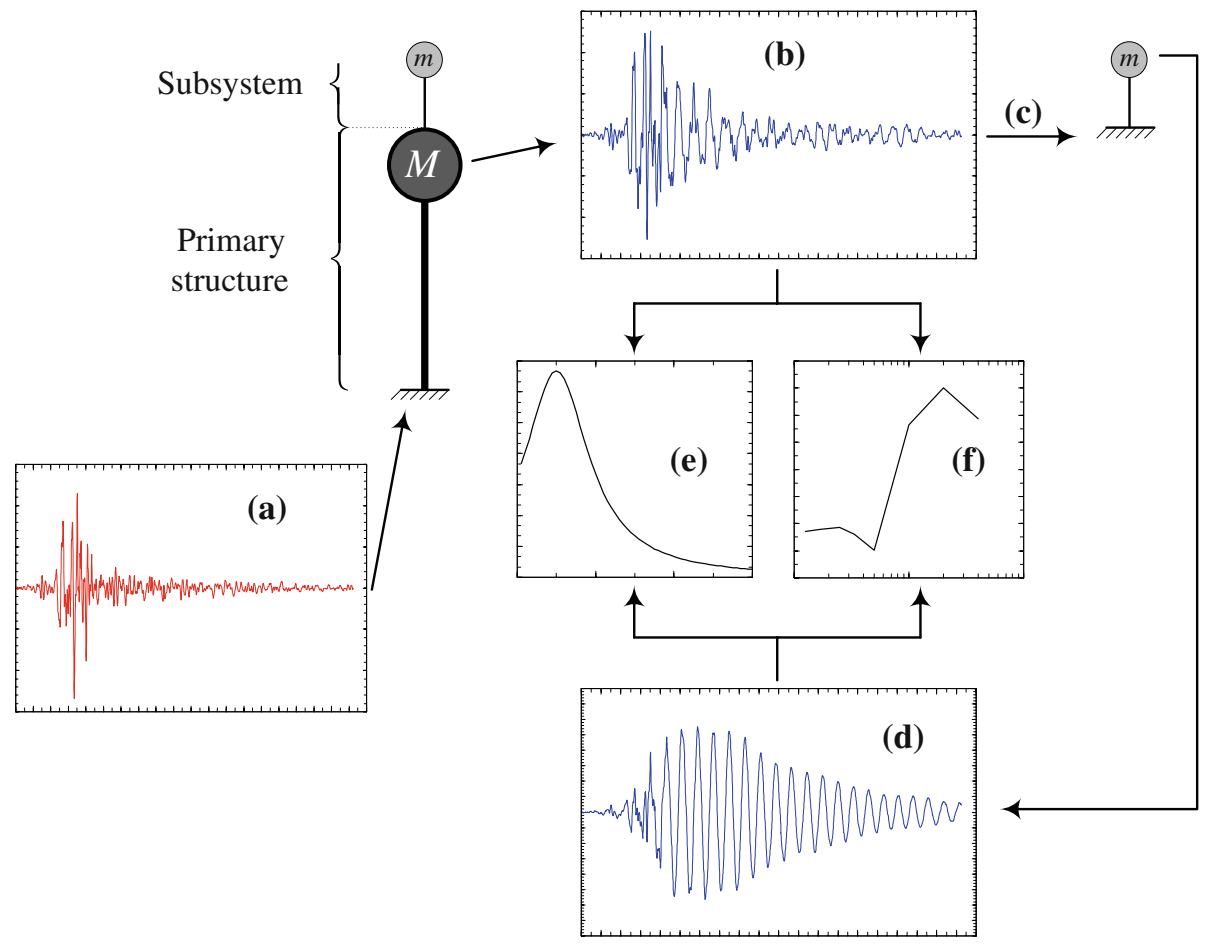

Fig. 7 Calculation procedure diagram. a 164 Registered ground acceleration time histories from the European Strong-Motion Data Base. b Acceleration (response) of the primary structure. $\mathbf{c}$ This acceleration is considered as a ground motion for the subsystem. $\mathbf{d}$ Acceleration (response) of the subsystem. e Obtained results plotted in terms of $\frac{a_{\mathrm{tot}, \mathrm{s}}}{a_{\mathrm{tot}, \mathrm{p}}}$ vs. $\frac{T_{\mathrm{S}}}{T_{\mathrm{p}}}$. f Obtained results plotted in terms of $\frac{a_{\mathrm{tot}, \mathrm{s}}}{a_{\mathrm{tot}, \mathrm{s} \text { elastic }}}$ vs. $T_{\mathrm{S}}$

Plot of resonance factor For seismic design of nonstructural components, Eurocodes (European Committee for Standardisation (CEN) 2003) require the consideration of an horizontal force $F_{\mathrm{a}}$ which depends on several factors such as seismic zone, soil and structural characteristics and a resonance factor depending on height of the system, $H$, and the height of location of subsystem, $z$. The resonance factor proposed by Eurocode 8 is defined as:

$$
f_{\text {res, Eurocode }}=\frac{3\left(1+\frac{z}{H}\right)}{1+\left(1-\frac{T_{\mathrm{s}}}{T_{\mathrm{p}}}\right)^{2}}-0.5
$$

$T_{\mathrm{S}}$ represents the initial natural subsystem period and $T_{\mathrm{p}}$ is the initial natural primary structure period. In this study resonance factor with a ratio $\frac{z}{H}=0$ is assumed, in order to compare Eq. 1 with the obtained results. Indeed, this study do not consider the ratio $\frac{Z}{H}$ as a parameter, hence $\frac{z}{H}=0$.

In this paper, the resonance factor is plotted as the ratio of the subsystem total acceleration over the primary structure total acceleration, $\frac{a_{\mathrm{tot}, \mathrm{s}}}{a_{\mathrm{tot}, \mathrm{p}}}$, versus the ratio of initial natural frequency of the primary structure over the subsystem natural frequency, $\frac{f_{\mathrm{p}}}{f_{\mathrm{s}}}=\frac{T_{\mathrm{s}}}{T_{\mathrm{p}}}$.

The average of maximum accelerations obtained from the whole earthquake database is calculated. In other words, one curve is given for each hysteretic model and for each strength reduction factor (Fig. 7e). 

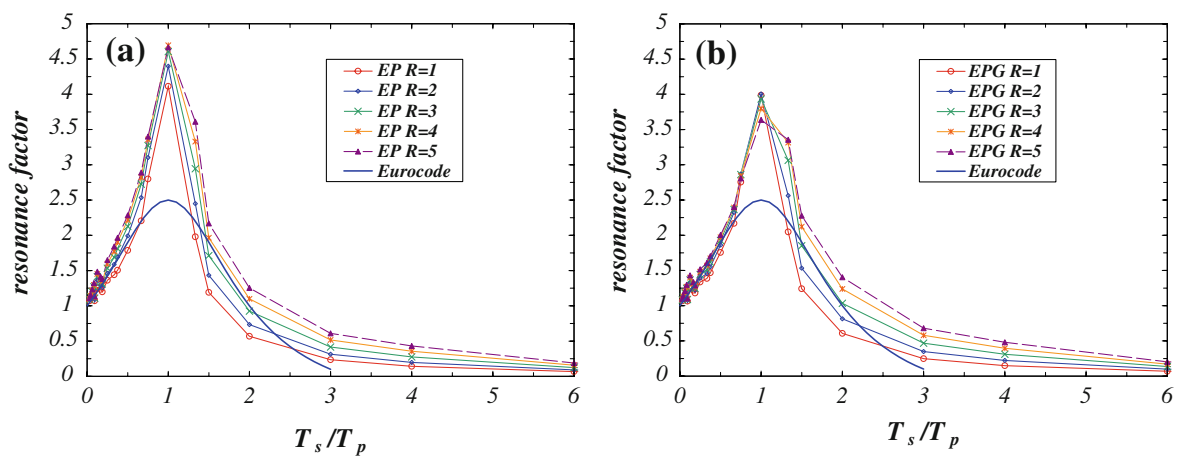

Fig. 8 Comparison between curves of resonance factor obtained (mean values) and curve given by Eurocode 8. a Elastoplastic model and Eurocode for $R=1,2,3,4$ and 5. b EPG-model and Eurocode for $R=1,2,3,4$ and 5

Plot of amplification factor The amplification factor is plotted in terms of the ratio of the subsystem total acceleration for an inelastic primary structure over the corresponding value for an elastic structure, $\frac{a_{\mathrm{tot}, \mathrm{s}}}{a_{\mathrm{tot}, \mathrm{s}_{\text {elastic }}}}$, versus the initial natural subsystem period, $T_{\mathrm{s}}=\frac{1}{f_{\mathrm{s}}}(\mathrm{Lin}$ and Mahin 1985).

The average of maximum accelerations obtained from the whole earthquake database is calculated. In other words, one curve is given for each hysteretic model and for each strength reduction factor (Fig. 7f).

\section{Results}

\subsection{Plot of resonance factor}

From the obtained results (Fig. 8; Table 1), the following conclusions are made:

- Even if the general shape of curve proposed by Eurocode 8 is confirmed, its peak value is significantly underestimated;

- The hysteretic models except for the elastoplastic model give a similar value for the resonance peak value (ratio $\frac{T_{\mathrm{s}}}{T_{\mathrm{p}}}=1$ ). Hence, in Fig. 8, only results using EP and EPG-model are shown;

- Using EPG-model, an increase in ductility decreases the resonance peak. The opposite is obtained using EP-model;

- Before the resonance peak value, EPG-model presents lower scattered values than EP-model;

- After the resonance peak value, EPG-model tends towards slightly higher values than EP-model;

- Using EP-model, curves computed with $R=5$ and $R=1$ may be considered as the higher bound and the lower bound, respectively;

- Using EPG-model, curves computed with $R=5$ and $R=1$ may be considered as the higher bound and the lower bound respectively, except in the neighborhood of the peak value. 
Table 1 Mean values at the peak of the curve of resonance, $\mu$, and their standard deviation, $\sigma$, for different models and strength reduction factor values, $R$

\begin{tabular}{|c|c|c|c|c|c|c|c|c|c|c|c|c|c|c|c|}
\hline \multirow[b]{2}{*}{ Model } & \multicolumn{3}{|c|}{$R=1$} & \multicolumn{3}{|c|}{$R=2$} & \multicolumn{3}{|c|}{$R=3$} & \multicolumn{3}{|c|}{$R=4$} & \multicolumn{3}{|c|}{$R=5$} \\
\hline & $\bar{\mu}$ & $\sigma$ & $\mu+\sigma$ & $\bar{\mu}$ & $\sigma$ & $\mu+\sigma$ & $\bar{\mu}$ & $\sigma$ & $\mu+\sigma$ & $\bar{\mu}$ & $\sigma$ & $\mu+\sigma$ & $\bar{\mu}$ & $\sigma$ & $\mu+\sigma$ \\
\hline EP & 4.11 & 1.26 & 5.37 & 4.40 & 1.21 & 5.61 & 4.61 & 1.35 & 5.96 & 4.69 & 1.51 & 6.20 & 4.67 & 1.60 & 6.26 \\
\hline EPG & 3.99 & 1.28 & 5.27 & 4.00 & 1.18 & 5.18 & 3.93 & 1.25 & 5.18 & 3.80 & 1.29 & 5.09 & 3.64 & 1.30 & 4.94 \\
\hline Q & 3.92 & 1.30 & 5.22 & 3.86 & 1.21 & 5.07 & 3.69 & 1.25 & 4.94 & 3.51 & 1.27 & 4.78 & 3.33 & 1.27 & 4.60 \\
\hline QZ & 3.96 & 1.29 & 5.25 & 3.84 & 1.19 & 5.03 & 3.70 & 1.22 & 4.92 & 3.54 & 1.25 & 4.79 & 3.38 & 1.26 & 4.64 \\
\hline Tak & 3.93 & 1.30 & 5.23 & 3.97 & 1.21 & 5.19 & 3.85 & 1.28 & 5.12 & 3.68 & 1.31 & 4.98 & 3.51 & 1.31 & 4.81 \\
\hline TakZ & 3.98 & 1.29 & 5.27 & 3.95 & 1.19 & 5.14 & 3.84 & 1.26 & 5.10 & 3.69 & 1.29 & 4.97 & 3.52 & 1.30 & 4.82 \\
\hline
\end{tabular}
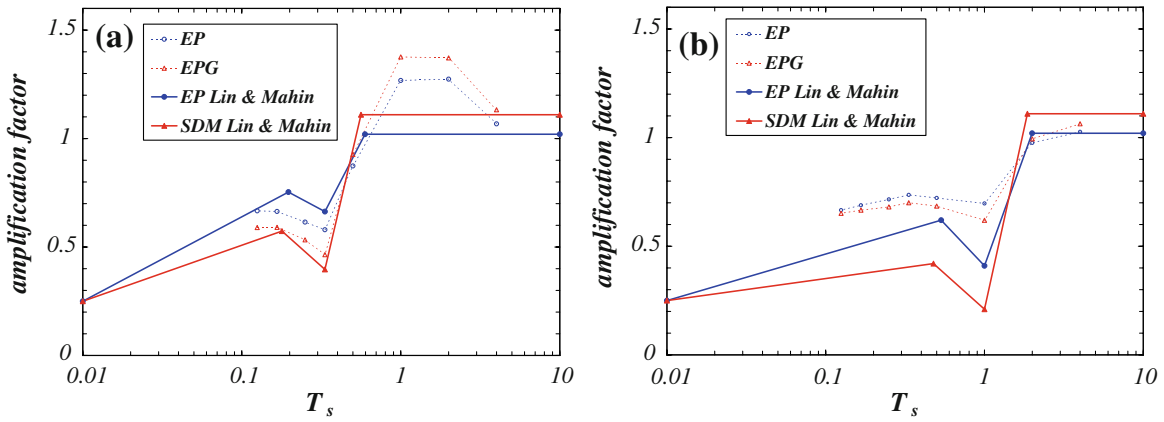

Fig. 9 Comparison between curves of amplification factor obtained with elastoplastic model and EPG-model and curves given by Lin and Mahin guidelines (strength reduction factor $R=4$ ). SDM is stiffness degradation model. a Primary structure period $T_{\mathrm{p}}=0.33 \mathrm{~s}$. b Primary structure period $T_{\mathrm{p}}=1 \mathrm{~s}$

\subsection{Plot of amplification factor}

From the obtained results (Fig. 9), the following conclusions are made:

- General shape of guidelines proposed by Lin and Mahin is realistic;

- Depending on the primary structure period, values proposed by Lin and Mahin may not correctly represent the obtained results. Indeed, for higher primary structure periods, Lin and Mahin overestimate the benefit of inelastic behavior (Fig. 9b);

- Amplification factor depends on strength reduction factor values.

For general purposes, a log-normal distribution of amplification factors obtained with the whole earthquake database considering strength reduction factor is assumed. In other words, one curve per model and per primary structure period is obtained and a mean and a standard deviation of this distribution are computed. It is observed that:

- The mean values of amplification factor (assuming a log-normal distribution) have a good agreement for all hysteretic models except for elastoplastic model. Hence, only results using EP-model and EPG-model are shown in Fig. 9;

- Arithmetic mean and log-normal mean of amplification factor are nearly identical for all cases. 

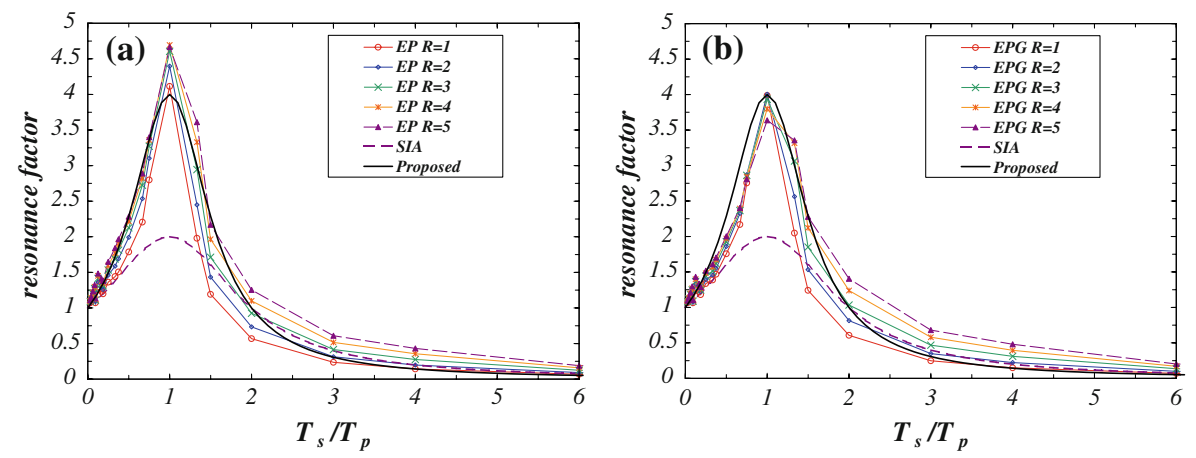

Fig. 10 Curves of resonance factor obtained and proposed. a Comparison between curve proposed and results considering elastoplastic model. b Comparison between curve proposed and results considering EPG-model

\subsection{Proposed plots}

\subsubsection{Proposed plot of resonance factor}

For nonstructural components, Swiss codes (Swiss Society of Engineers and Architects (SIA) 2003) require the consideration of an horizontal force $F_{\text {a }}$ composed of two factors $f_{\text {seismic }}$ and $f_{\text {res }}$. The seismic factor $f_{\text {seismic }}$ depends on seismic zone, soil and structural characteristics and a height term. $f_{\text {res }}$ is a resonance factor depending on the ratio of the periods $\frac{T_{\mathrm{s}}}{T_{\mathrm{p}}}$ :

$$
f_{\text {res, SIA }}=\frac{2}{1+\left(1-\frac{T_{\mathrm{s}}}{T_{\mathrm{p}}}\right)^{2}}
$$

This expression can be modified to fit the results:

$$
f_{\text {res, proposed }}=\frac{4}{1+3\left(1-\frac{T_{\mathrm{s}}}{T_{\mathrm{p}}}\right)^{2}}
$$

Figure 10 compares obtained plot of resonance factor (EP-model and EPG-model) to the plot proposed in the literature (European Committee for Standardisation (CEN) 2003). Note that the proposed curve is not dependant of the strength reduction factor $R$.

\subsubsection{Proposed plot of amplification factor}

In order to generalize the applicability of obtained results, plots based on Lin and Mahin (1985) are proposed. Figure 11 compares plots of amplification factor obtained to those proposed in the literature for four values of primary structure period. Table 2 gives amplification factor values for the different primary structure periods studied.

\section{Conclusions}

The behavioral characteristics of light subsystems supported on primary structures which may yield during a ground motion have been analyzed in this paper. Values for acceleration ratios 

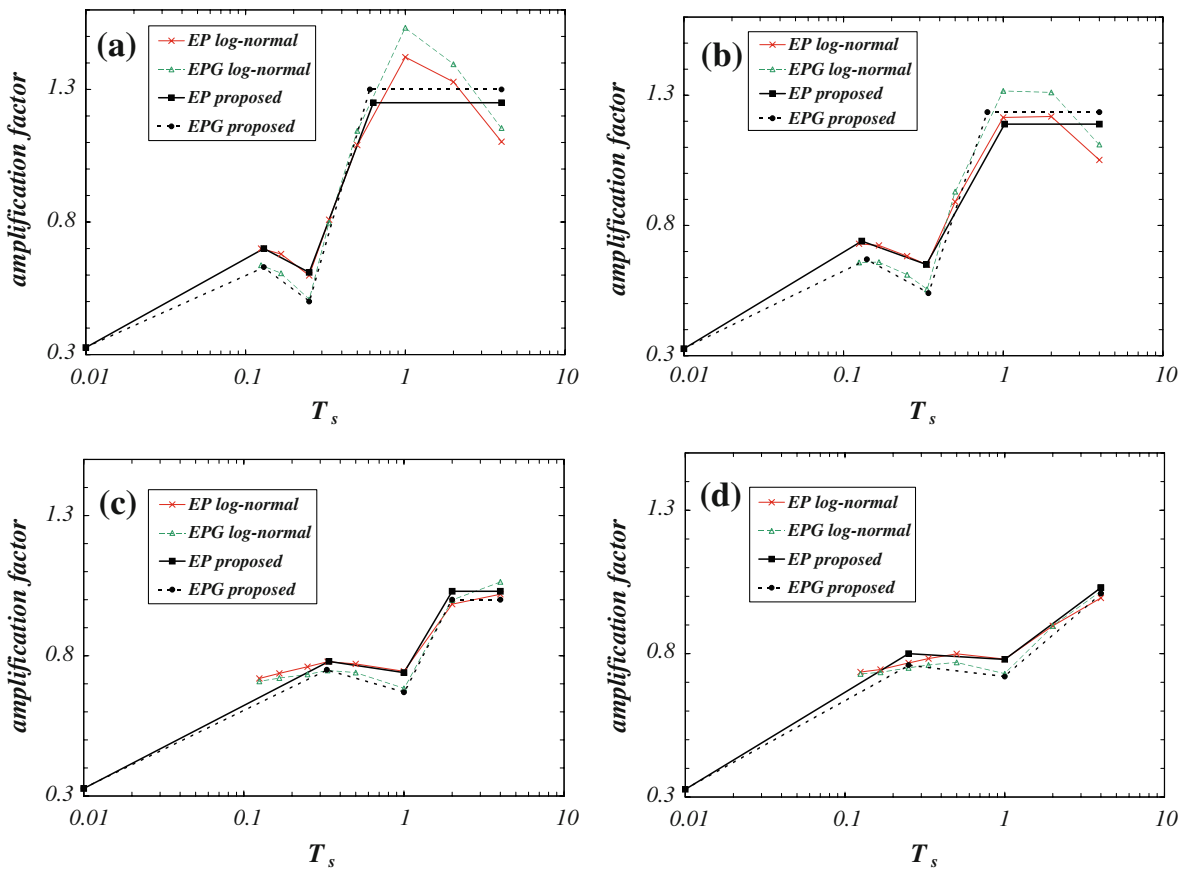

Fig. 11 Curves of amplification factor obtained and proposed based on elastoplastic model and EPG-model. a Primary structure period $T_{\mathrm{p}}=0.25 \mathrm{~s}$. b Primary structure period $T_{\mathrm{p}}=0.33 \mathrm{~s}$. c Primary structure period $T_{\mathrm{p}}=1 \mathrm{~s}$. d Primary structure period $T_{\mathrm{p}}=1.33 \mathrm{~s}$

Table 2 Amplification factor values proposed, $f_{\mathrm{a}}$, for elastoplastic model, EPG-model and different primary structure periods, $T_{\mathrm{p}}$

\begin{tabular}{llllllllllll}
\hline & \multicolumn{1}{c}{ EP } & \multicolumn{1}{c}{ EPG } \\
\hline \multirow{2}{*}{$T_{\mathrm{p}}=1.33 \mathrm{~s}$} & $T_{\mathrm{S}}$ & 0.01 & 0.25 & 1 & 4 & 4 & 0.01 & 0.25 & 1 & 4 & 4 \\
& $f_{\mathrm{a}}$ & 0.33 & 0.80 & 0.78 & 1.03 & 1.03 & 0.33 & 0.76 & 0.72 & 1.01 & 1.01 \\
$T_{\mathrm{p}}=1 \mathrm{~s}$ & $T_{\mathrm{S}}$ & 0.01 & 0.34 & 1 & 2 & 4 & 0.01 & 0.33 & 1 & 2 & 4 \\
& $f_{\mathrm{a}}$ & 0.33 & 0.78 & 0.74 & 1.03 & 1.03 & 0.33 & 0.75 & 0.67 & 1 & 1 \\
$T_{\mathrm{p}}=0.67 \mathrm{~s}$ & $T_{\mathrm{S}}$ & 0.01 & 0.22 & 0.50 & 1.96 & 4 & 0.01 & 0.23 & 0.5 & 1.99 & 4 \\
& $f_{\mathrm{a}}$ & 0.33 & 0.78 & 0.73 & 1.09 & 1.09 & 0.33 & 0.73 & 0.66 & 1.12 & 1.12 \\
$T_{\mathrm{p}}=0.5 \mathrm{~s}$ & $T_{\mathrm{S}}$ & 0.01 & 0.17 & 0.50 & 1.30 & 4 & 0.01 & 0.21 & 0.50 & 1 & 4 \\
& $f_{\mathrm{a}}$ & 0.33 & 0.74 & 0.70 & 1.10 & 1.10 & 0.33 & 0.71 & 0.60 & 1.10 & 1.10 \\
$T_{\mathrm{p}}=0.33 \mathrm{~s}$ & $T_{\mathrm{S}}$ & 0.01 & 0.13 & 0.33 & 1.02 & 4 & 0.01 & 0.14 & 0.34 & 0.80 & 4 \\
& $f_{\mathrm{a}}$ & 0.33 & 0.74 & 0.65 & 1.19 & 1.19 & 0.33 & 0.67 & 0.54 & 1.24 & 1.24 \\
$T_{\mathrm{p}}=0.25 \mathrm{~s}$ & $T_{\mathrm{S}}$ & 0.01 & 0.13 & 0.25 & 0.63 & 4 & 0.01 & 0.13 & 0.25 & 0.60 & 4 \\
& $f_{\mathrm{a}}$ & 0.33 & 0.70 & 0.61 & 1.25 & 1.25 & 0.33 & 0.63 & 0.5 & 1.30 & 1.3 \\
\hline
\end{tabular}

are proposed as design guidelines for subsystems by varying strength reduction factor, natural frequencies of vibration and considering different hysteretic models and using 164 recorded ground acceleration time histories from the European Strong-Motion Database (Ambraseys et al. 2002; Lestuzzi et al. 2004, 2007).

In general, results are independent of hysteretic models, except for elastoplastic model. Therefore, results can be classified in two groups : elastoplastic model and stiffness degrading models (EPG-model, Q-model and Takeda model). Obtained results are expressed in plots of 
amplification factor and resonance factor for these hysteretic models. Differences are found between existing curves and obtained curves. Therefore new values are proposed.

Values of amplification factor proposed by Lin and Mahin are compared to those obtained in this study. Proposed values permit a better estimation of inelastic behavior impact in terms of acceleration with an elastic analysis only are obtained, particularly for structures with higher values of primary structure periods. Indeed, values proposed by Lin and Mahin overestimate the benefit from inelastic behavior of the primary structure if its period is high.

Obtained results of resonance factor highlight an underestimation of peak value of resonance factor when using current design codes. In order to simplify the use of these results, a new formulation of resonance factor based on Swiss Codes (Swiss Society of Engineers and Architects (SIA) 2003) are defined. This curve has a similar formulation as Eurocode 8, therefore it is easily applicable. Note that the effect of the parameter $\frac{z}{H}$ was not assessed.

The reported results of the considered study were gained with limited range of parameter's values. Therefore, some extensions are needed in order to validate and to generalize the proposed values. Some possible extensions of the study are discussed below

Resonance factor Equation 3 may be improved to include ductility term (strength reduction factor) for primary structure. For instance the strength reduction factor may be incorporated in Eq. 3. Moreover, it is possible to propose two different formulations of resonance factor depending on the hysteretic model (elastoplastic model and degradation stiffness such as EPG-model).

Amplification factor To improve proposed values, a larger period range needs to be considered. In fact, lower and higher $\frac{T_{\mathrm{s}}}{T_{\mathrm{p}}}$ values have not been studied. This larger period range will complete the curve.

Mean of amplification factor with the log-normal distribution gives nearly the same values than an arithmetic mean. However, standard deviation may be important $\left(\sigma_{\max } \approx 0.5\right)$. Therefore, it may be necessary to define curves of amplification factor for each strength reduction factor to reduce scattering in these values.

Finally, to improve the shape of the curve of amplification factor, a supplementary point for a subsystem period $T_{\mathrm{s}} \approx 0.1 \mathrm{~s}$ may be considered.

\section{References}

Allahabadi R, Powell GH (1988) Drain-2DX user guide. Report No. UCB/EERC-88/06. College of Engineering, University of California, Berkeley

Ambraseys N, Smit P, Sigbjornsson R, Suhadolc P, Margaris B (2002) Internet-site for european strongmotion database (ESD). European Commission, Research-Directorate General, Environment and Climate Programme. http://www.isesd.cv.ic.ac.uk/ESD/frameset.htm

Chaudhuri SR, Villaverde R (2008) Effect of building nonlinearity on seismic response of nonstructural components: a parametric study. ASCE J Struct Eng 134(4):661-670

Chen Y, Soong TT (1988) State-of-the-art review: seismic response of secondary systems. Eng Struct 10(4):218-228

Chopra A (2001) Dynamics of structures: theory and applications to earthquake engineering. ISBN: 0-13086973-2. Prentice Hall, Englewood-Cliffs

European Committee for Standardisation (CEN)(2003) Design of structures for earthquake resistance. European prestandard prEN 1998-1:2003 Eurocode 8. In: European Standards

Favez P (2007) Spectre de plancher et comportement non-linéaire. Semester project, École Polytechnique Fédérale de Lausanne, EPFL

Kingston KM (2004) An evaluation of floor response spectra for acceleration-sensitive nonstructural components supported on regular frame structures. Master's thesis, University of Maryland

Lestuzzi P, Badoux M (2003a) An experimental confirmation of the equal displacement rule for RC structural walls. In Proceedings of the fib-symposium: concrete structures in seismic regions, Paper no 127. Athens, Greece, 6-8 May 2003 
Lestuzzi P, Badoux M (2003b) The $\gamma$-model: a simple hysteretic model for reinforced concrete walls. In Proceedings of the fib-symposium: concrete structures in seismic regions, Paper no 126. Athens, Greece, 6-8 May 2003

Lestuzzi P, Belmouden Y, Trueb M (2007) Nonlinear seismic behaviour of structures with limited hysteretic energy dissipation capacity. Bull Earthq Eng 5(4):549-569

Lestuzzi P, Schwab P, Koller M, Lacave C (2004) How to choose earthquake recordings for nonlinear seismic analysis of structures. In: 13th World Conference on Earthquake Engineering

Lin J, Mahin S (1985) Seismic response of light subsystems on inelastic structures. ASCE. J struct eng 111(2):400-417

Litton RW (1975) A contribution to the analysis of concrete structures under cyclic loading. PhD thesis, University of California, Berkeley

Otani S (1974) Inelastic analysis of R/C frame structures. J Struct Div 100(7):1433-1449

Saatcioglu M (1991) Modeling hysteretic force-deformation relationships for reinforced concrete elements. Am Concr Inst (ACI-SP) 127:153-198

Saiidi M, Sozen MA (1981) Simple nonlinear seismic analysis of rc structures. ASCE J Struct Div (Proc Am Soc Civil Eng) 107(5):937-953

Schwab P, Lestuzzi P (2007) Assessment of the seismic non-linear behaviour of ductile wall structures due to synthetic earthquakes. Bull Earthq Eng 5(1):67-84

Swiss Society of Engineers and Architects (SIA) (2003) Actions on structures, SIA261:2003. SN 505 261. In Swiss Standards

Takeda T, Sozen MA, Nielsen NN (1970) Reinforced concrete response to simulated earthquakes. ASCE J Struct Div (Proc Am Soc Civil Eng) 96(12):2557-2573

Triou P (2006) Influence de la non linéarité sur les spectres de plancher. Master's thesis, École Polytechnique Fédérale de Lausanne, EPFL 